\title{
Analyse De La Capacité De L'Etat Burundais à Répondre Aux Besoins Fondamentaux de la population: Cas De L'eau Et De La Santé
}

\begin{abstract}
Ndabarushimana Alexis, PhD
Docteur en sociologie du développement rural,

Directeur Chargé de la Formation Diplômante et de la Recherche à l'Ecole Nationale d'Administration / Burundi et Coordinateur des Conférences Publiques de l'ENA, Vice-Président du Réseau des Ecoles Nationales d'Administration d'Afrique (RENA_AFRIQUE) Enseignant-Chercheur de sociologie du développement rural à l'Université Espoir d'Afrique et de Management Public à l'ENA
\end{abstract}

Doi: 10.19044/esj.2018.v14n33p80 URL:http://dx.doi.org/10.19044/esj.2018.v14n33p80

\begin{abstract}
Access to water and health care is one of the basic needs of the human being. This study conducted with 96 individuals selected using Alain BOUCHARD's technic highlights the state of play on the capacity of the State of Burundi to meet the basic needs of the population in the water and health sectors. The study also shows the level of satisfaction of public service users in these sectors. The respondents were distributed in 26 localities in 8 provinces of Burundi. The results of this research shows that Burundi is still unable to effectively meet the needs of the population in the water and health sectors. And this despite the implementation of the policy of free health care for pregnant women and children under 5 years since 2006. Only 51.15\% say they are satisfied by the health services offered. The environmental sanitation and drinking water supply had been integrated into the Minimum Care Package (MSP), but the results obtained in this study show that the road ahead is still long. Only $7.8 \%$ of respondents are very satisfied in urban areas while in rural areas $12.6 \%$ are very satisfied. The satisfaction rate is $70.92 \%$ in urban areas compared to $45.04 \%$ in rural areas. In urban areas, $21.28 \%$ are not satisfied compared to $42.36 \%$ in rural areas.
\end{abstract}

Keywords: Basic needs, Drinking water, Health care, Sanitation, satisfied

\section{Résumé}

L'accès à l'eau et aux soins de santé font partie des besoins fondamentaux de la population. Cette étude menée auprès de 96 individus retenus en utilisant la formule d'Alain BOUCHARD met en lumière l'état 
des lieux sur la capacité de l'Etat burundais à répondre aux besoins de la population dans les secteurs de l'eau et de la santé. L'étude montre aussi le niveau de satisfaction des usagers des services publics dans ces secteurs. Les enquêtés étaient répartis dans 26 localités de 8 provinces du Burundi. L'ensemble des résultats de cette recherche permet de retenir que l'Etat burundais n'arrive pas encore à répondre efficacement aux besoins des populations dans les secteurs de l'eau et de la santé. Et ce malgré la mise en œuvre de la politique de gratuité des soins de santé pour les femmes enceintes et les enfants de moins de 5ans depuis 2006. Seulement 51,15\% affirment être satisfaits par les services de santés offerts. L'assainissement du milieu et l'approvisionnement en eau potable avait été intégré dans le Paquet Minimum de Soins (PMS), mais les résultats obtenus dans cette étude montrent que le chemin à parcourir reste encore long. Seulement $7,8 \%$ d'enquêtés sont très satisfait en milieu urbain alors qu'en milieu rural $12,6 \%$ sont très satisfaits. Le taux de satisfaction représente $70,92 \%$ en milieu urbain contre $45,04 \%$ en milieu rural. En milieu urbain, c'est $21,28 \%$ qui ne sont pas satisfaits contre $42,36 \%$ en milieu rural.

Mots clés: Besoins fondamentaux, Eau potable, Soins de santé, Assainissement, satisfait

\section{Introduction}

L'accès à l'eau et aux soins de santé de qualité constitue un droit humain fondamental selon la Déclaration Universelle des Droits de l'Homme (DUDH, 1948). C'est la possibilité de l'homme à satisfaire ces besoins qui témoigne, en partie, de la décence dans sa vie.

Aujourd'hui, les politiques nationales se réfèrent aux exigences des Objectifs de Développement Durable (ODD). Le 6 6 ème ODD vise à "garantir l'accès de tous à l'eau et à l'assainissement et assurer une gestion durable des ressources en eau'. Quant au $3^{\text {ème, }}$ il vise à "permettre à tous de vivre en bonne santé et promouvoir le bien-être de tous à tout âge".

De Miras et Le Tellier (2005) signalaient déjà qu'en Afrique, les proportions de la population n'accédant pas aux services d'Alimentation en Eau Potable (AEP) représentaient en 2005, 38\% contre 19\% en Asie et 15\% en Amérique latine et Caraïbe. Celles n'accédant pas à l'assainissement représentaient en Afrique $40 \%$ contre $52 \%$ en Asie et $22 \%$ en Amérique latine et Caraïbe. Dans l'article publié par Brou et al. (2018), nous lisons que selon l'OMS (2010) environ 1,1 milliard de personnes n'avaient pas accès à une eau potable en 2010 et 2,4 milliards ne disposaient pas de systèmes d'assainissement adéquats. 
En ce qui concerne le Burundi, sa constitution, en son article 52, stipule que «Toute personne est fondée à obtenir la satisfaction des droits économiques, sociaux et culturels indispensables à sa dignité et au libre développement de sa personne, grâce à l'effort national et compte tenu des ressources du pays ».

La constitution du Burundi est compatible avec les conventions internationales sur la nécessité d'appliquer le principe du citoyen avant tout. Ce principe vise à rendre les agents de l'Etat beaucoup plus redevables aux usagers des services publics. C'est sous-entendu une protection sociale globale qui peut être définie comme «un ensemble de mesures publiques ou privées qui visent à réduire la pauvreté et les vulnérabilités économiques et sociales » (MFPTE, 2013). Cette définition qui revient dans la «stratégie de la protection sociale au Burundi» adopté en janvier 2015, implique un ensemble de mesures qui concourent à assurer à la population une sécurité minimale en termes de revenus, à faciliter l'accès aux services de base et à aider les ménages à mieux gérer les risques auxquels ils sont confrontés.

Le gouvernement Burundais vient de mettre en place le Plan National de Développement (PND) 2018-2027. Ce plan qui s'étend sur une période de dix ans s'inspire toujours de la vision 2025.

La vision 2025 est un instrument de guidage et d'orientation qui aide le gouvernement à atteindre ses objectifs dont la finalité est la satisfaction de l'intérêt général. Par ailleurs, dans la concrétisation de la Vision 2025, le Cadre Stratégique de croissance et de Lutte Contre la Pauvreté, deuxième génération (CSLP II) ainsi que le Programme National pour la Réforme Administrative (PNRA) ont étés adoptés par le Gouvernement en 2012.

Néanmoins, bien qu'un certain nombre de politiques positives qui visent la satisfaction des besoins humains de base aient été formulées, les résultats obtenus dans la mise en œuvre des politiques et la prestation de services révèlent des lacunes en matière de satisfaction des usagers. C'est dans ce cadre que l'évaluation sur terrain constitue une nécessité pour juger du niveau de satisfaction dans les secteurs de l'eau et de la santé.

Dans ce contexte de prise de conscience de la qualité de réponses à apporter aux populations, la question d'accès à ces services de base reste problématique et se pose dans le sens de responsabilité, de transparence et de redevabilité en tant que valeurs qui doivent caractériser tous les services publics burundais.

Mais alors, quelle est la capacité de l'Etat burundais à répondre aux besoins fondamentaux de la population?

En effet, quels sont les différents instruments élaborés par l'Etat burundais pour répondre aux besoins fondamentaux de la population?

Quel est le niveau de satisfaction des usagers des services publics dans ces secteurs? 
L'objectif principal de cet article est d'analyser la capacité de l'Etat burundais à répondre aux besoins fondamentaux de la population en matière de l'eau et de soins de santé. En d'autres termes, cet article permet de saisir les différents instruments élaborés par l'Etat burundais pour répondre aux besoins fondamentaux de la population dans les secteurs de l'eau et de la santé et de montrer le niveau de satisfaction des usagers des services publics dans ces secteurs.

\section{Concepts et méthodologie \\ Concept}

Etant toujours redevable envers les populations, le pouvoir public doit assurer l'accès des populations aux besoins fondamentaux, notamment, l'accès à l'eau potable et aux soins de santé.

En effet, les conflits cycliques qu'a connu le Burundi dans son histoire explique sa situation socio-économique actuelle qui le classe parmi les pays à faible capacité à répondre aux besoins des populations en matière d'eau et santé, deux éléments constitutifs des "besoins fondamentaux" des populations. Ainsi, le concept de besoins fondamentaux dans cet article mérite d'être défini. Avec une faible augmentation de la production, la demande croissante des biens de consommation et la forte demande en eau et en soins de santé, la pauvreté a tendance à s'accroître touchant en grande partie les populations rurales. Selon l'OAG (2012), "Il faut mettre en cause la période d'instabilité politique qui a fait que le PIB diminue de 3\% après 1993 et cela jusqu'en 2001'' (p. 30).

Selon l'Organisation Mondiale de la Santé (OMS), une eau potable est une eau que l'on peut boire sans risque pour la santé. L'OMS la définit comme une eau ayant les caractéristiques microbiennes, chimiques et physiques qui répondent aux directives de l'OMS ou aux normes nationales relatives à la qualité de l'eau de boisson.

En clair, "une eau potable est une eau qui ne contient pas d'agents pathogènes ou d'agents chimiques à des concentrations pouvant nuire à la santé ». (http://www.la-cde.com/fr/espace-client/espace-sensibilisation/105eau-oms.html: visité le 06/02/2018 à $16 \mathrm{~h} 28 \mathrm{~min}$ ).

Afin de définir précisément une eau potable, des normes ont été établies qui fixent notamment les teneurs limites à ne pas dépasser pour un certain nombre de substances nocives et susceptibles d'être présentes dans l'eau. L'OMS précise aussi que le fait qu'une eau soit conforme aux normes, c'est-à-dire potable, ne signifie donc pas qu'elle soit exempte de matières polluantes, mais que leur concentration a été jugée suffisamment faible pour ne pas mettre en danger la santé du consommateur.

Selon ces normes, une eau potable doit être exempte de germes pathogènes (se dit d'un organisme capable de provoquer une maladie: 
bactéries, virus) et d'organismes parasites, car les risques sanitaires liés à ces micro-organismes sont grands. Elle ne doit contenir certaines substances chimiques qu'en quantité limitée : il s'agit en particulier de substances qualifiées d'indésirables ou de toxiques, comme les nitrates et les phosphates, les métaux lourds, ou encore les hydrocarbures et les pesticides pour lesquelles des concentrations maximales admissibles ont été définies.

A l'inverse, la présence de certaines substances peut être jugée nécessaire comme les oligo-éléments indispensables à l'organisme. C'est dans cet optiques que l'Observatoire Régionale de l'environnement (ORE) (2016), rappelait qu'à la fin du XIXème siècle, Louis Pasteur confirmait déjà que "nous buvons 90\% de nos maladies".

Une eau potable doit aussi être une eau agréable à boire : elle doit être claire, avoir une bonne odeur et un bon goût. Pour avoir bon goût, il lui faut contenir un minimum de sels minéraux dissous (de 0,1 à 0,5 gramme par litre), lesquels sont par ailleurs indispensables à l'organisme. Cette définition que l'OMS donne à l'eau potable montre bien que la qualité de l'eau est liée à celle de la santé. Ainsi, l'accès à l'eau potable permet d'améliorer la qualité de la santé de la population.

L'OMS (1946) définit la santé comme étant 'un état de bien-être complet physique, mental et social, et ne consiste pas seulement en une absence de maladie ou d'infirmité '. Cette définition met en lien la qualité de l'eau et la satisfaction des besoins fondamentaux.

\section{Méthodologie \\ Population d'enquête}

La population cible est constituée de la population locale bénéficiaire des services publics en eau et en soins de santé (Fonctionnaires, commerçants, fermiers, chômeurs, travailleurs indépendants, étudiants, etc.). Et les enquêtés ciblés étaient uniquement les chefs de ménage.

Ainsi, selon le Recensement Général de la Population et de l'Habitat (RGPH, 2008) les ménages faisant partie de notre population d'enquête répartis dans les huit provinces concernées par l'étude sont au nombre de 843279 habitants.

Selon Mucchielli (1991), on appelle population d'enquête, « l'ensemble du groupe humain concerné par les objectifs de l'enquête C'est dans cet univers que sera découpé l'échantillon » (p. 16). C'est aussi, selon N'da (2002), "un ensemble d'unités élémentaires (une personne, un groupe, une ville, un pays) qui partagent des caractéristiques communes précises selon des critères définis" (p. 65). Quant à Aktouf (1992), la population d'enquête comme se définit comme étant «l'ensemble indifférencié des éléments parmi lesquels seront choisis ceux sur qui s'effectueront les observations» (p.72). La population constitue l'ensemble des sujets sur 
lesquels devrait porter la recherche. Chaque individu de la population est appelé sujet ou unité statistique.

Compte tenu des spécificités de notre étude, nous souscrivons à l'idée de Ghiglione, \& Matalon (1978) selon laquelle : "il est inutile d'interroger toute la population de l'étude,'

(p. 29).

\section{Technique d'échantillonnage}

Citant Borg (1971), Munyerere (2015), mentionne que 'l'échantillonnage est une technique qui permet de choisir un nombre limité d'individus, d'objets ou d'événement comme des sujets qui représentent la population cible', (p.59).

Pour concevoir un échantillon pouvant fournir des estimations statistiquement fiables, nous avons dû choisir une taille d'un échantillon quantitatif nécessaire pour assurer la représentativité des enquêtés. Elle a été calculée à travers la formule d'Alain BOUCHARD.

Le tableau ci-dessous nous montre la détermination de la taille de l'échantillon selon Bouchard.

Tableau 1: Détermination de la taille de l'échantillon

\begin{tabular}{|c|c|c|c|c|c|c|c|c|c|}
\hline \multirow{5}{*}{$\begin{array}{l}\text { Taille de la } \\
\text { population }\end{array}$} & \multicolumn{9}{|c|}{ Précision } \\
\hline & \multicolumn{3}{|c|}{90 fois sur 100} & \multicolumn{3}{|c|}{95 fois sur 100} & \multicolumn{3}{|c|}{99 fois sur 100} \\
\hline & $\# 177$ & \#177; & $\# 177$; & $\# 177$; & $\# 177$; & $\# 177$; & $\# 177$; & $\# 177$; & $\# 177$; \\
\hline & $10 \%$ & 5 & $1 \%$ & 10 & $5 \%$ & 1 & 10 & $5 \%$ & $1 \%$ \\
\hline & & $\%$ & & $\%$ & & $\%$ & $\%$ & & \\
\hline Infini & 68 & 271 & 6.765 & 96 & 384 & 9.601 & 166 & 664 & 16.589 \\
\hline 1.000 .000 & 68 & 271 & 6.720 & 96 & 384 & 9.513 & 166 & 663 & 16.319 \\
\hline 100.000 & 68 & 270 & 6.336 & 96 & 383 & 8.763 & 166 & 659 & 14.229 \\
\hline 50.000 & 68 & 269 & 5.959 & 96 & 381 & 8.057 & 165 & 655 & 12.459 \\
\hline 10.000 & 67 & 263 & 4.035 & 95 & 370 & 4.899 & 163 & 622 & 6.239 \\
\hline 5.000 & 67 & 257 & 2.875 & 94 & 357 & 3.288 & 161 & 586 & 3.842 \\
\hline 1.000 & 63 & 213 & 871 & 88 & 278 & 905 & 142 & 399 & 943 \\
\hline 500 & 60 & 176 & 466 & 81 & 217 & 475 & 125 & 285 & 485 \\
\hline 100 & 41 & 73 & 99 & 49 & 80 & 99 & 63 & 87 & 99 \\
\hline 50 & 29 & 42 & 50 & 33 & 44 & 50 & 39 & 47 & 50 \\
\hline
\end{tabular}

Source : Bouchard A, cité par Munyerere (2015)

Selon la formule d'Alain Bouchard cité par Munyerere (2015), « quand la population faisant l'objet d'étude est inférieure ou égale à 1.000.000 d'individus, on la fait correspondre à un échantillon de 96 individus avec une 
marge d'erreur de $10 \% »($ p.50). C'est donc à partir de la formule suivante que nous avons pu obtenir un échantillon représentatif.

$$
\begin{gathered}
n c=\frac{n}{1+\frac{n}{N}} \quad \text { En développant, la formule devient : } n c=\frac{n}{1+\frac{n}{N}}=\frac{n}{\frac{N+n}{N}}=\frac{n}{1} \times \frac{N}{N+n} \\
n c=\frac{n \times N}{N+n}
\end{gathered}
$$

$\mathrm{N}=$ Total de la population-mère (population cible)

$\mathrm{nc}=$ Echantillon corrigé.

L'échantillon total universel d'un univers fini est $n=96$

Echantillon corrigé (nc)

Les 843279 chefs de ménages sont répartis comme suit :

Tableau2 : Répartition de la population d'enquête par province

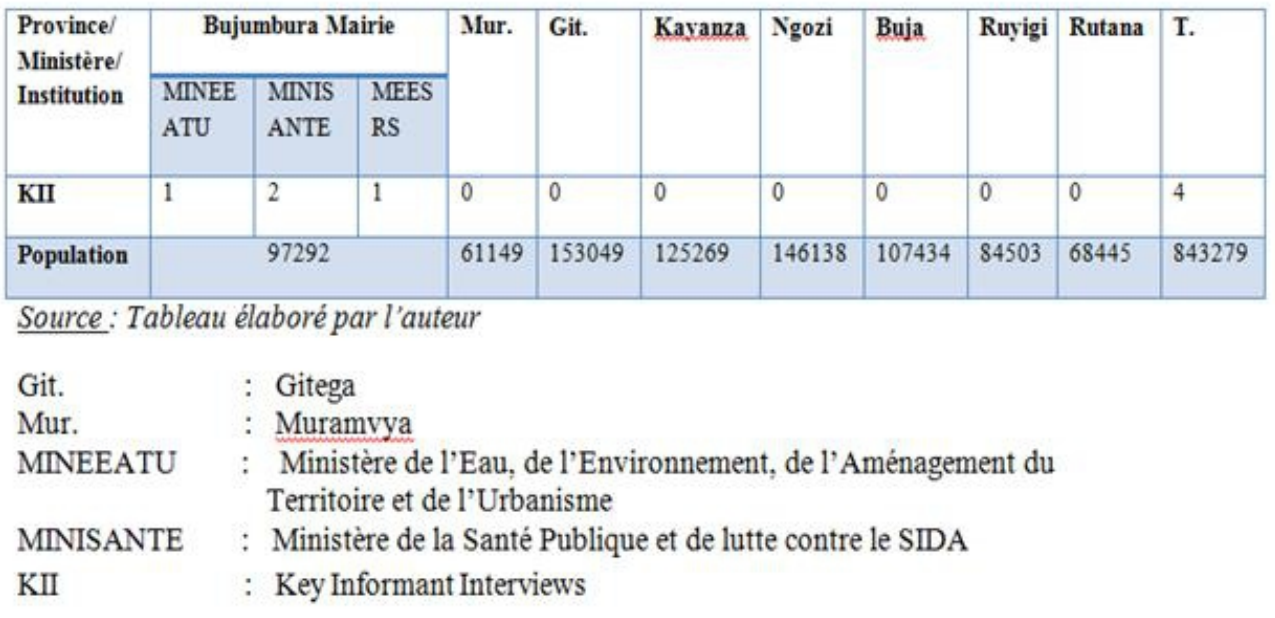

Ainsi, en appliquant la formule d'Alain BOUCHARD, l'échantillon total corrigé $n c$ de notre étude est la suivante :

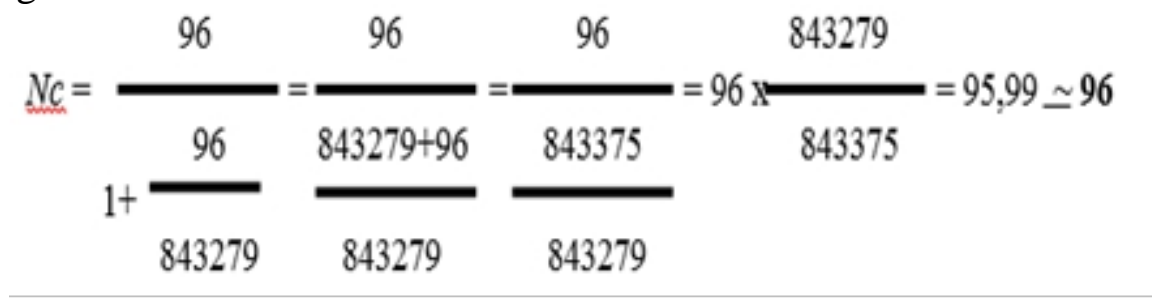

Avec cette formule, la taille de l'échantillon total s'élève à 96 individus en tenant compte des vingt-six (26) localités visitées dans 8 provinces avec 4 informateurs clés interviewés. Partant d'un échantillon choisis de 96 individus dans un univers fini de 843279 ménages, nous avons réparti 
proportionnellement les échantillons dans chaque strate représentée par une province.

Ainsi, les échantillons retenus par strate sont répartis dans le tableau 3 de la page suivante.

Tableau3 : Répartition de l'échantillon par province

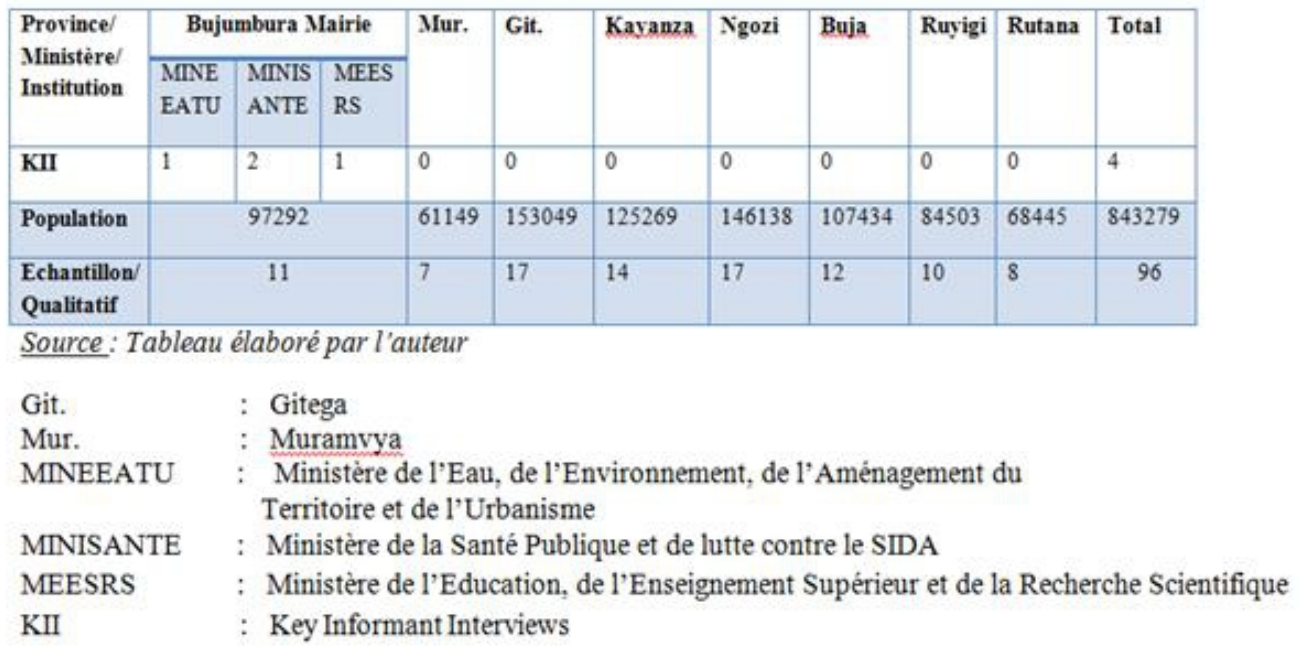

Ainsi, les données du tableau 3 montrent que les trois ministères concernés directement par l'objet de notre étude ont été tous représentés à raison de 1 individu interviewé au Ministère de l'Environnement, de l'eau, de l'Aménagement du Territoire et de l'Urbanisme. Au Ministère de la Santé Publique et de Lutte contre le SIDA, nous avons interviewé 2 individus.

Les quatre individus retenus pour l'interview sont tous de la Mairie de Bujumbura auxquels s'ajoutent 11 individus qui ont complété notre questionnaire d'enquête. Les 85 autres enquêtés qui ont complété notre questionnaire sont répartis dans 7 autres provinces à savoir Muramvya, Gitega, Kayanza, Ngozi , Bujumbura, Ruyigi et Rutana.

\section{Différents instruments élaborés par l'Etat burundais pour répondre aux besoins fondamentaux de la population}

Sur le plan institutionnel, le Burundi a déjà mis en place un certain nombre de textes régissant l'organisation et la gestion des biens et des services publics. Mais malgré l'existence de ces textes et la promulgation de quelques lois, les questions de transparence, de lutte contre la corruption, les procédures de suivi et évaluation, de planification axée sur la performance, l'implémentation des systèmes de gestion et de contrats de performance rencontrent des limites qui ont motivé la recherche dans ce secteur. 


\section{Vision nationale}

Au Burundi, toute planification s'inspire de la vision 2025 qui sert de boussole dans l'orientation de l'activité économique. Le Cadre Stratégique de Croissance et de Lutte contre la Pauvreté (CSLP) découle lui-même de cette vision nationale. Aussi, le cadre de dépenses et les plans stratégiques existent dans tous les ministères.

Après l'évaluation du CSLP II, le Plan National de Développement du Burundi, (PND BURUNDI 2018-2027) vient d'être mis en place. Son objectif à long terme vise à rétablir les équilibres structurels de l'économie burundaise. Le PND 2018-2027 constitue un outil d'orientation stratégique de référence pour les différents départements ministériels. Ces derniers devront s'y référer pour construire leurs politiques et plans d'actions. Le PND est élaboré dans un environnement politique, économique et social caractérisé par l'ouverture au dialogue et à la concertation entre le Gouvernement et les partenaires des partis politiques, de la société civile, des confessions religieuses et de la jeunesse qui sont des acteurs incontournables du développement.

Dans le cadre de la mise en œuvre du Plan National de Développement, trois objectifs stratégiques ont été identifiés pour ce faire: (i) améliorer l'accès aux services d'eau potable ; (ii) améliorer l'accès aux services d'assainissement de base et (iii) renforcer la gestion des sous-secteurs eau potable et assainissement de base.

Dans le secteur de la santé, le Plan National de Développement préconise l'amélioration du cadre légal en faveur de la santé de la population et la mise en oeuvre des interventions sanitaires, particulièrement celles touchant des groupes les plus vulnérables notamment les femmes enceintes et les enfants de moins de cinq ans, les personnes vivant avec le VIH, les personnes vivant avec un handicap, les personnes âgées, les différentes catégories sociales minoritaires (orphelins, etc....). La mise en œuvre de ce programme se fera à travers les projets suivants : (i) amélioration de la demande de soins et services de santé et (ii) renforcement de la complémentarité et des synergies intersectorielles ; (iii) military specific program for VIH (MSP-HIV) (appui à l'HMK) ; (iv) projet de mise en à réseau des laboratoires de santé dans l'EAC.

Dans son Plan National de Développement Sanitaire (2011-2015), le Ministère de la santé publique et de lutte contre le SIDA souligne qu'au Burundi, la situation sanitaire reste préoccupante et marquée par la prédominance de nombreuses maladies transmissibles et non transmissibles.

Selon l'annuaire statistique (2009), les maladies qui constituent les principales causes de morbidité et de mortalité sont le paludisme, les infections respiratoires aigües, les maladies diarrhéiques, la malnutrition, le VIH/Sida et la tuberculose. De nombreux facteurs de risque liés aux conditions de vie plus particulièrement en rapport avec l'hygiène, l'assainissement, l'alimentation 
et l'environnement contribuent à accroître la charge de morbidité dans la population. Les groupes vulnérables tels que les femmes enceintes, les enfants et les orphelins sont particulièrement affectés. Cette situation est encore aggravée par la faible protection sociale de la population face au risque de maladie.

Un autre instrument élaboré par le gouvernement est le Programme d'Actions Prioritaires, 2018. Ce programme définit trois objectifs stratégiques à savoir: (i) améliorer l'accès aux services d'eau potable ; (ii) améliorer l'accès aux services d'assainissement de base ; (iii) améliorer la gestion des sous- secteurs eau potable et assainissement de base.

Ce programme vise la mise en cuvre des projets suivants: (i) amélioration du cadre légal des secteurs d'eau potable et d'assainissement de base ; (ii) réforme de la REGIDESO pour le développement des services d'eau potable en milieu urbain; (iii) amélioration de la planification et de coordination des sous-secteurs d'eau potable et d'assainissement de base ; (iv) appui à la maîtrise d'ouvrage communal en matière de planification, développement et gestion professionnelle des infrastructures d'AEPA ; (v) réorganisation et déconcentration des services de l'Agence burundaise de l'Hydraulique et de l'Assainissement en Milieu Rural (AHAMR) afin d'être plus proche des structures communales et (vi) renforcement du rôle du maître d'ouvrage de la commune dans la gestion et maintenance des AEP.

Le Ministère de la santé publique et de la lutte contre le SIDA précise, dans son Programme d'Action Prioritaire 2011-2015, qu' "à l'horizon de l'an 2027, la Couverture Sanitaire Universelle (CSU) sera possible grâce à l'établissement d'un système de santé et de soins performant, fort et résilient ainsi que d'un système social d'assurance maladie basé sur des initiatives publiques et privées, individuelles et collectives, accessible et équitable, capable de satisfaire le droit à la santé à tous et en particulier des groupes les plus vulnérables à travers le développement d'un partenariat solide et interagissant au niveau national, régional et international »(p.55). Mais l'existence d'un cadre légal à lui seul ne suffit pas, il nécessite l'instauration d'un leadership qui permet d'assurer l'accès aux besoins fondamentaux.

Ainsi, l'Etat burundais, reste responsable de services publics dans le secteur de l'eau comme précisé dans le code de l'eau en vigueur au Burundi en son article 111: "La mobilisation, la production, le transport et la distribution de l'eau potable en vue de satisfaire les besoins du public, constituent de services publics industriel et commercial sous la responsabilité de l'Etat. Des mécanismes de régulation et de délégation de service public de l'eau mis en place par l'autorité compétente conformément à la législation en vigueur', (p. 43). 


\section{Mesures législatives d'anti- corruption}

Au Burundi, il existe le code pénal qui s'applique à tous sans oublier les autres mesures de bonne gestion. Au Ministère de la Santé Publique et de la Lutte contre le Sida par exemple il a été mis en place des comités anticorruption à tous les niveaux de la pyramide sanitaire.

Toutes les structures mises en place ont les capacités nécessaires pour remplir leurs responsabilités. Au niveau du gouvernement, il y a un soutien et une volonté politique de soutien au programme anti-corruption à travers la politique de tolérance zéro, la mise en place de la cour et de la brigade anticorruption et l'Inspection Générale de l'Etat. Ces structures sont capables de faire respecter la loi car des cas de corruption ont été déjà traités depuis leur mise en place.

Selon l'Office des Nations Unies Contre la Drogue et le Crime (UNODC), (2015), le travail de la cour anticorruption est alimenté par les dossiers en provenance de la Brigade Anti-Corruption et de l'Inspection Générale de l'Etat. Le Parquet près la Cour peut également être saisi par dénonciation. Etant donné sa compétence spécialisée, elle reçoit aussi les dossiers dessaisis des juridictions de droit commun.

Selon la même organisation, en avril 2011, la Cour anticorruption a communiqué les données suivantes aux examinateurs de l'application par le Burundi des articles 15 à 42 du Chapitre III («Incrimination, détection et répression ») et des articles 44 à 50 du Chapitre IV («Coopération internationale») de la Convention des Nations Unies contre la corruption 2010 - 2015: “il y avait 453 affaires prononcées depuis la création de la Cour, dont 350 définitives et 103 encore avant faire droit. Il y avait 593 affaires inscrites en tout. 102 affaires avaient étéfrappées d'appel, 27 affaires avaient bénéficié d'un acquittement et 140 affaires étaient encore en litige'. (p. 15). Aujourd'hui, des sommes importantes non encore publiées officiellement ont été récupérées des mains des auteurs de la corruption.

\section{Procédures de suivi et évaluation}

$\mathrm{Au}$ sein de l'administration burundaise, il existe des cellules qui se chargent du suivi et évaluation. Au ministère de l'environnement on l'appelle "atelier d'évaluation". Toutefois, cet atelier n'est régi par aucune loi. Il ne relève que de l'organisation interne du ministère. C'est pour cette raison que cette forme d'organisation n'existe pas dans d'autres ministères.

$\mathrm{Au}$ sein des Ministères où il y a des plans d'actions, on instaure un système d'évaluation annuelle des plans d'action. En outre, au sein de certains ministères comme le MSPLS, il est organisé trimestriellement des 
ateliers d'évaluation des réalisations à tous les niveaux de la pyramide sanitaire. Et le système d'évaluation touche aussi les secteurs de l'eau et de la santé.

\section{Satisfaction des usagers du service public en matière d'accès à l'eau potable \\ Graphique 1 : Niveau de satisfaction des usagers en approvisionnement en eau potable}

Niveau de satisfaction des usagers en approvisionnement en eau

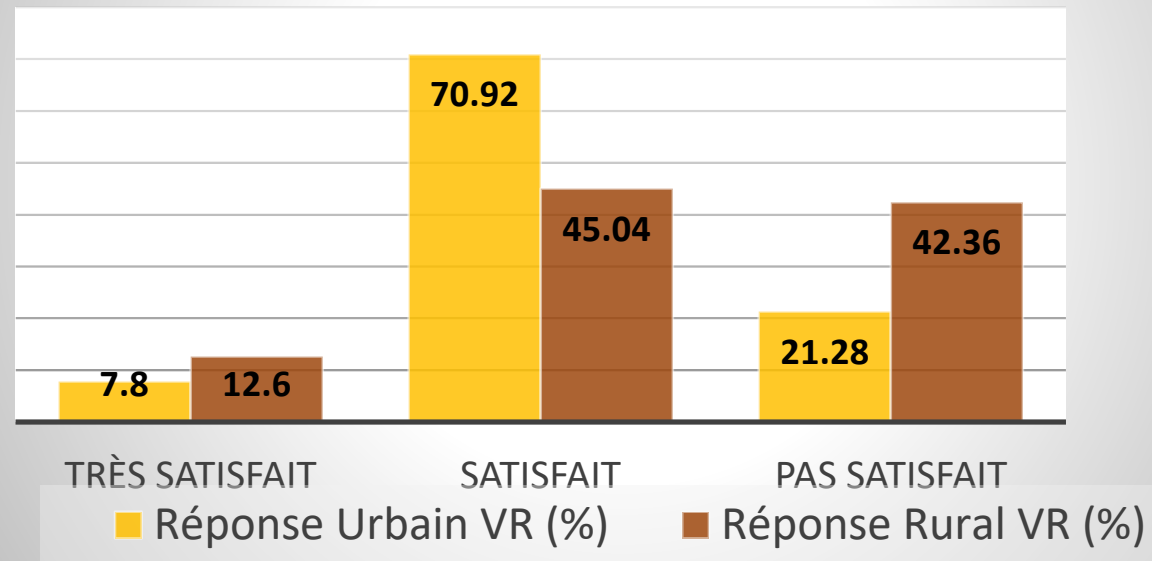

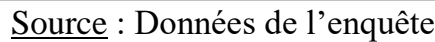

De la graphique 1, il ressort qu'en milieu urbain, c'est seulement 7,8\% d'enquêtés qui sont très satisfait en milieu urbain alors qu'en milieu rural ceux qui sont très satisfaits représentent $12,6 \%$. Le taux de satisfaction représente $70,92 \%$ en milieu urbain contre $45,04 \%$ en milieu rural. En milieu urbain, c'est $21,28 \%$ qui ne sont pas satisfaits contre $42,36 \%$ en milieu rural. Ces écarts s'expliquent par des coupures d'eau répétitives en milieu urbain où les fontaines publiques sont rarement alimentées. Néanmoins, comme beaucoup de ménages possèdent des robinets chez eux, ils expriment une grande satisfaction.

Cette situation montre donc que la politique nationale d'eau n'est pas effective dans tout le pays. Ce qui requiert de l'administration un déploiement d'efforts supplémentaires dans la mise en œuvre de cette politique.

Nos résultats montrent que les populations, rurale et urbaine éprouvent, à des degrés différents, des difficultés à accéder à l'eau potable du fait que ceux qui sont très satisfaits restent peu nombreux dans les deux milieux. Cette réalité avait été relevée par le Ministère de l'Eau, de l'Environnement, de 
l'Aménagement du Territoire et de l'Urbanisme (2012) quand il élaborait la stratégie nationale de l'eau (2011-2020). Dans cette stratégie, nous lisons ceci: "les efforts du gouvernement burundais dans la réforme et le développement du secteur de l'eau et de l'assainissement menés au cours de la décennie internationale de l'eau potable et de l'assainissement ont été bloqués par la crise socio-politique de 1993'. (p. 2).

Le même ministère (2009) a réaffirmé cela en 2009 dans le document de "politique nationale de l'eau" en disant que "suite à la crise qui a éclaté en 1993, les taux de desserte en eau et de couverture en assainissement de base ont fortement chuté. En milieu rural, le taux de desserte est passé de 70\% en 1992 à 43\% en 2005(...). En milieu urbain, le taux de desserte est passé de 93\% en 1992 à 75,8\% en 1999'. (pp.33-34).

Nos résultats ne s'écartent pas donc de cette réalité car nous avons trouvé qu'en milieu rural, les populations continuent à consommer de l'eau des rivières et des puits non potable avec un taux d'accès à l'eau potable inférieur à celui du milieu urbain.

\section{Satisfaction des usagers des services publics en matière d'accès aux soins} de santé

Graphique 2 : Satisfaction des bénéficiaires par rapport aux services de santé

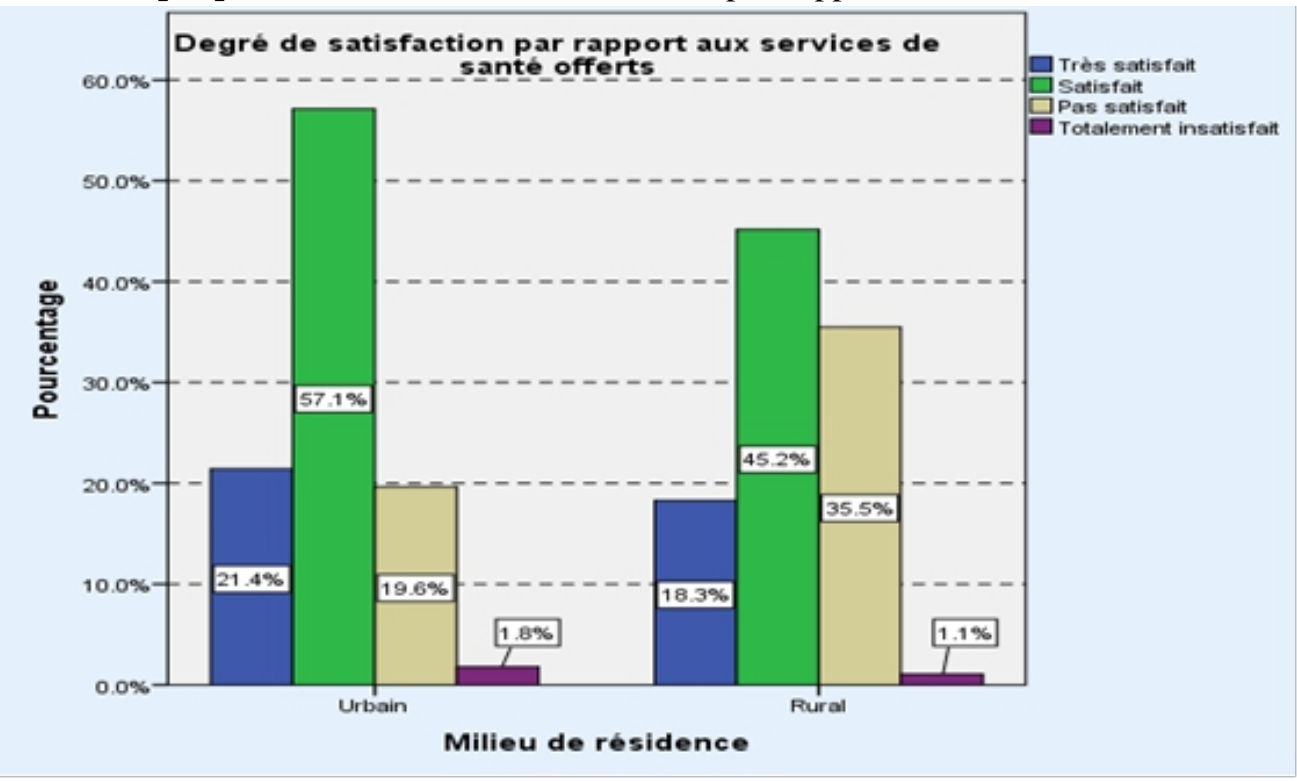

Source : Données de l'enquête

Les données de la graphique 2 montrent qu'en milieu urbain, les enquêtés très satisfaits en matière d'accès aux soins de santé représentent $21 \%$. Ceux qui sont satisfaits représentent $57.1 \%$ tandis que ceux qui ne sont pas 
satisfaits représentent $19.6 \%$. Cependant, il existe $1.8 \%$ d'enquêtés qui sont totalement insatisfaits.

En milieu rural, les enquêtés très satisfaits en matière d'accès aux soins de santé représentent $18.3 \%$. Ceux qui sont satisfaits représentent $45.2 \%$ tandis que ceux qui ne sont pas satisfaits représentent 35.5\%. Dans certaines provinces, il existe aussi des cas d'insatisfaction totale qui représentent $1.1 \%$.

En effet, les enquêtés satisfaits sont ceux qui reconnaissent être bien reçu par les agents de santé indépendamment de la longue duré d'attente.

Ceux qui ne sont pas satisfaits déplorent le manque d'équipements médicaux, le manque d'ambulance pour assurer le transfert des patients vers d'autres structures sanitaires. Ces situations occasionnent des dépenses supplémentaires pour les patients ou les membres de la famille du patient.

En combinant les données du milieu rural et du milieu urbain en termes de satisfaction, on trouve que les enquêtés très satisfait en matière d'accès aux soins de santé représentent une moyenne de $19.65 \%$ au Burundi. Ceux qui sont satisfaits représentent une moyenne de $51.15 \%$ tandis que ceux qui ne sont pas satisfaits représentent une moyenne de $25.55 \%$. La moyenne de ceux qui sont totalement insatisfaits est de $1.45 \%$.

Dans son rapport de synthèse sur l'étude sur le financement de la santé au Burundi réalisée en 2014, le ministère de la santé publique et de lutte contre le SIDA (2004) souligne que le système de santé au Burundi est largement sous-financé et connait une grande dépendance vis-à-vis du financement extérieur. A titre illustratif, selon le même rapport, la Dépense Totale de Santé (2012) est de 26 US\$ per capita alors que l'OMS recommande 44 US\$. La part de la Santé dans les Dépenses Publiques est en-deçà de $9 \%$, comparé à l'Engagement de la Déclaration d'Abuja qui est de 15\%. Les dépenses des régimes publics sont financées à hauteur de $26,7 \%$ par les revenus nationaux tandis que $73,3 \%$ viennent des transferts d'origine étrangère (p.17). Cette réalité trouve sa justification dans les propos de Djimouko (2016) qui se référait à son mémoire de DEA en géographie de la santé présenté en 2008. Cet auteur fait remarquer qu'en Afrique subsaharienne, le secteur de la santé a été tenu, pendant longtemps, à l'écart des programmes de développement. Seuls les secteurs agricole et industriel étaient privilégiés.

$\mathrm{Au}$ Burundi, le financement de la protection sociale en santé est fragmenté avec la coexistence de plusieurs régimes assurantiels : Carte d'Assistance Médicale (CAM) pour le secteur informel et rural ; Gratuité couplée au Financement Basé sur la Performance (Gratuité-FBP) ; Régime des Indigents ; Mutuelles Communautaires de Santé (MCS) ; Mutuelle de la Fonction Publique (MFP) et Assurances privées, mutuelles de santé des caféiculteurs. Cette fragmentation se traduit par un manque de cohérence induisant quelques fois des situations paradoxales notamment l'introduction 
de la CAM « rénovée »versus MCS. Elle est également source de double payement (Gratuité versus MFP) et de surcoûts de transaction.

La plupart des régimes assurantiels connait un sous-financement chronique. Le décalage entre, d'une part, le taux de couverture (approximatif) de la population par ces régimes qui est grossièrement estimé à près de $50 \%$, et d'autre part, le poids de ces mécanismes dans la dépense courante totale de santé qui est de $17,1 \%$ illustre bien ce sous-financement. Celui-ci provoque, à son tour, l'accumulation d'arriérés de paiements ou créances sur les factures émises au titre de ces mécanismes dans les formations sanitaires (FOSA) publiques, particulièrement les hôpitaux et dans cette situation, il est quasi impossible d'avoir accès aux soins de santé de qualité.

Nous estimons que le problème majeur repose sur le fait que la mutualité au Burundi implique une petite partie de la population. Nous proposons à cet effet que toute la population soit mobilisée et réunie au sein d'une mutuelle nationale et unique où chaque membre de la communauté contribue à hauteur de mille franc burundais (1000 fbu) mensuellement. En le faisant, on peut identifier des personnes volontaires qui prendraient en charge les contributions des indigents. Dans ces circonstances, on peut estimer qu'une somme importante peut être affectée dans le secteur de la santé et par conséquent, améliorer la qualité des soins de la population. Aussi, la mise en place d'un système d'assurance maladie obligatoire (AMO) permettra-t-elle d'améliorer la qualité des soins et d'éviter le gaspillage que l'on observe dans certains ménages.

\section{Discussion des résultats}

$\mathrm{Au}$ regard de ces données, il est important de signaler que les prévisions du CSLP II en matière de l'accès à l'eau potable ne pouvaient pas être atteintes sans la mise en œuvre effective de la politique nationale d'eau. D'autres contraintes d'ordre institutionnel, démographique, socioéconomique limitent la réalisation des objectifs définis par le CSLP II.

En effet, ce dernier envisage une augmentation du taux d'accès à l'eau potable de $85 \%$ en 2009 à $95 \%$ en 2015 en milieu urbain et de $50 \%$ à $70 \%$ en milieu rural pendant la même période, ce qui est difficile à réaliser.

Le sixième Objectif de Développement Durable (ODD) stipule qu'il faut, jusqu'en 2030 « Garantir l'accès de tous à l'eau et à l'assainissement et assurer une gestion durable des ressources en eau ». Mais au regard des résultats de notre analyse, on se rend compte que le chemin à parcourir reste encore long : par exemple au Burundi jusqu'aujourd'hui, c'est seulement 3.0\% de Burundais qui possèdent des robinets familiaux, $35.1 \%$ utilisent toujours l'eau des rivières contre $30.2 \%$ qui utilisent l'eau des fontaines publiques. Ces résultats montrent qu'il existe des inégalités dans l'accès à l'eau potable selon le milieu de résidence et coïncident donc avec ceux de De Miras, C. et Le 
Tellier, J. (2005) qui ont montré que l'accès à l'eau potable des pauvres des quartiers défavorisés au Maroc reste en-dessous des objectifs fixés.

Nous souscrivons aussi aux propos de ces auteurs selon lesquels " les Objectifs du Millénaire, en énonçant l'exigence d'une réduction de moitié du nombre de personnes n'ayant pas accès à l'eau potable en 2015, ont mis la barre très haut à l'égard de responsables politiques et gestionnaires confrontés, au Maroc et dans bien d'autres pays, à des problèmes de différents ordres auxquels ils tentaient de faire face depuis de nombreuses années". (p.11).

Nos résultats montrent que les populations, rurale et urbaine éprouvent, à des degrés différents, des difficultés à accéder à l'eau potable. Cette réalité avait été relevée par le Ministère de l'Eau, de l'Environnement, de l'Aménagement du Territoire et de l'Urbanisme (2012) quand il élaborait la stratégie nationale de l'eau (2011-2020). Cette stratégie souligne que: "les efforts du gouvernement burundais dans la réforme et le développement du secteur de l'eau et de l'assainissement menés au cours de la décennie internationale de l'eau potable et de l'assainissement ont été bloqués par la crise socio-politique de 1993'. (p. 2).

Le même ministère (2009) a réaffirmé cela en 2009 dans le document de "politique nationale de l'eau' en disant que "suite à la crise qui a éclaté en 1993, les taux de desserte en eau et de couverture en assainissement de base ont fortement chuté. En milieu rural, le taux de desserte est passé de $70 \%$ en 1992 à 43\% en 2005(...). En milieu urbain, le taux de desserte est passé de $93 \%$ en 1992 à 75,8\% en 1999', (pp.33-34). Nos résultats ne s'écartent pas donc de cette réalité car nous avons trouvé qu'en milieu rural, les populations continuent à consommer de l'eau des rivières et des puits non potable avec un taux d'accès à l'eau potable inférieur à celui du milieu urbain. Ces résultats coïncident aussi avec ceux obtenus par la direction générale des publications de presse burundaise (2011) qui ont montré que la plupart des 225 puits du Burundi se concentrent dans les provinces Kirundo et Ruyigi avec seulement $15 \%$ de puits en état de fonctionnement où dans $19 \%$ de cas la qualité de l'eau a été évaluée comme sale.

Dans le secteur de la santé, nos résultats ne s'éloignent pas de ceux présentés par l'OIT dans son rapport de 2014 sur l'évaluation technique pour la mise en place d'un Socle de protection sociale au Burundi selon lesquels environ $17 \%$ des personnes malades n'auraient pas accès aux soins de santé et $81,5 \%$ des patients seraient obligés de s'endetter ou de vendre des biens afin de faire face aux dépenses liées à la santé. Cette réalité observée dans la société burundaise n'est pas différente de celle qu'observait déjà Boyodi (2016) chez les populations du centre du Togo où les résultats de son étude montrent que les populations rurales présentent des difficultés majeures d'accès aux soins chirurgicaux du fait de leur coût relativement élevés. 
Au Burundi, le secteur de la santé est coordonné par le Ministère de la santé publique et de lutte contre le SIDA, qui a comme mission première de veiller à établir les conditions favorables à l'amélioration de la qualité de santé de la population. A cet effet, le milieu rural reste le plus exposé aux maladies à cause du manque des équipements.

Pour montrer les écarts entre le milieu rural et le milieu urbain, le Ministère de l'Eau, de l'Environnement, de l'Aménagement du Territoire et de l'Urbanisme (2013) précisait déjà qu' "en milieu rural, le service est exclusivement autonome et se base beaucoup plus sur l'auto-investissement des ménages malgré quelques projets de construction de latrines. En milieu urbain, le système autonome coexiste dans certains cas avec un réseau d'égouts géré par les services techniques municipaux, qui ne dessert qu'une toute petite partie de la population urbaine ». (p.20).

En effet, les résultats obtenus dans cette étude montrent que toutes les populations burundaises n'ont pas encore un accès facile aux services de santé. $28 \%$ d'enquêtés parcourent de 6 à $50 \mathrm{~km}$ pour atteindre une structure de soins, publique ou privée. C'est seulement $72 \%$ qui parcourent entre 1 et $5 \mathrm{~km}$. Ainsi, ces résultats coïncident avec ceux de l'OAG (2012) qui montraient déjà que "plus de $80 \%$ des ménages au moins vivent à près de $5 \mathrm{~km}$ d'un centre de santé, bien qu'il y ait des disparités remarquables au niveau de quelques provinces. Même s'il y a des progrès au niveau des infrastructures, le faible pouvoir d'achat des ménages et du coût élevé des soins de santé font que tout le monde ne peut être soigné. Le prix des services de santé au cours d'un mois par personne est estimé à 3.000 Fbu en milieu rural. En milieu urbain, il est estimé à 6.700 Fbu en moyenne. (...) en Mairie de Bujumbura, il est estimé à 8.000Fbu', (p.32).

Dans son étude menée en juin 2012 sur la situation économique du Burundi à la veille de la célébration du cinquantième anniversaire, l'OAG (2012 : 31) précisait déjà que les enfants de moins de 5 ans figurent parmi les catégories les plus touchées par des maladies et la mortalité. Le taux de mortalité infantile était de 101 décès pour 1000 naissances vivantes en 2000 et de 114 en 2007 (RGPH, 2008). Et comme le disait Ibrahima (2015), les causes de la dégradation de la santé humaine sont à rechercher dans l'environnement familial et d'une manière générale dans le milieu de vie. Dans son étude, Ibrahima (2015) a confirmé l'hypothèse selon laquelle 'l'état sanitaire moyen d'une population dépend de son niveau d'assainissement et d'équipement en infrastructures de base dont elle bénéficie, mais également de ses capacités d'adaptation (réactions et pratiques) aux contraintes '. Au vu de nos résultats, nous souscrivons nous aussi à cette réalité. C'est dans cette même logique que Brou (2018) affirmait que la santé physique des hommes découle de la santé de leur environnement. 
Une année avant la publication de Brou (2018), Ziad (2017) a souligné que les droits à l'eau et à l'assainissement ne sont isolés des autres droits de l'homme, et un lien étroit unit les individus qui ne jouissent pas des droits à l'eau et à l'assainissement et ceux ne bénéficiant pas non plus des droits au logement, à l'alimentation, à l'éducation et à la santé. L'accès à l'eau potable est une condition préalable à la jouissance d'un certain nombre de de droits de l'homme, comme le droit à l'éducation, le logement, la santé, la vie le travail et la protection contre les traitements cruels ou inhumains.

En outre, les résultats d'une enquête réalisée par Médecins sans frontière (2004) sur "l'accès aux soins de santé au Burundi », montre que l'accès aux soins de santé au Burundi reste problématique. En effet, selon les résultats de cette enquête, le système de recouvrement des coûts exclut presque un million de personnes des soins de santé au Burundi. En effet, avec un tel système, $17.4 \%$ des personnes malades n'ont pas accès aux soins, essentiellement faute d'argent (pour $81.7 \%$ d'entre eux). Même parmi les patients qui pensent être gravement malades, $14.5 \%$ ne vont pas à la consultation. Parmi les 14.5\%, 90.7\% n'y vont pas par manque d'argent (p.6). Les difficultés d'accès aux soins de santé ont été aussi observées par Doubogan, Y. O., Badou, S. A. O. \& Atchouta R. A. (2017) dans la société béninoise et ont mentionné que le coût d'accès élevé aux soins de santé modernes combiné à la pauvreté des ménages constitue un facteur majeur à l'accessibilité aux soins. De fait, les inégalités

d'accès aux soins et services de santé pour les ménages constituent un problème central en matière de santé publique.

Pour faire face à ce genre de situation de morbidité, de mortalité élevés et de non accès aux soins de santé, le taux de couverture vaccinale a été maintenu à plus de $90 \%$ pour les principaux antigènes. Cette réalité justifie une fois de plus le bon choix du gouvernement qui a mis en œuvre la politique de la gratuité des soins des moins de 5 ans et des femmes enceintes.

\section{Conclusion}

La présente étude a contribué à une meilleure connaissance de la capacité de l'Etat burundais à répondre aux besoins de la population dans les secteurs de l'eau et de la santé et à mettre en lumière le niveau de satisfaction des usagers des services publics dans ces secteurs. Il a fourni des informations utiles aux responsables des structures de l'eau et de la santé qu'elles soient publiques ou privées, ainsi qu'aux décideurs politiques qui ont le devoir de veiller à ce que toutes les couches sociales arrivent à satisfaire les besoins fondamentaux.

Les ressources humaines, financières et physiques inadéquates et l'insuffisance des infrastructures bien équipées restent des défis majeurs que le gouvernement burundais doit relever. 
Dans ce sens, les évaluations annuelles organisées par le Ministère de l'intérieur permettent aux autorités locales de gérer leur localité avec rigueur.

Dans les services publics burundais, la culture de rendre compte tend aussi à se développer suite aux recommandations émises par le gouvernement visant à faire en sorte que chaque ministère se dote d'un plan stratégique. Abordant l'aspect législatif, cette étude a montré que l'Etat burundais a mis en place un certain nombre de structures spécialisées dans la lutte contre la corruption.

Par rapport à l'accès à l'eau potable, nos résultats ont révélé que l'écart est très grand en milieu urbain et en milieu rural. En milieu urbain, la majorité des ménages utilisent l'eau fournie par la REGIDESO et 77,1\% en possèdent dans leurs maisons.

En milieu rural, plus de $88 \%$ des interviewés utilisent l'eau des rivières, des fontaines publiques ou des puits communautaires et dans certaines localités, les fontaines publiques ne sont pas alimentées de façon permanente. Ce manquement amène les familles à utiliser 1'eau non potable puisée dans les marais ou rivières. De telles situations Expliquent la forte morbidité au sein de la population d'enquête. Le rapport du Programme National Intégré de Lutte contre le Paludisme (2010) montre que le taux de morbidité au Burundi est passé de 36,23\% en 2005 à 34,07 en 2010 avec un taux de mortalité dans les hôpitaux de 39,55\% en 2005 à 34,07\% en 2010 . Cette situation est réelle dans un contexte où l'accès aux soins de santé et aux médicaments reste problématique.

En milieu rural, le niveau de satisfaction des populations est de $45.04 \%$ tandis qu'il est de $70.92 \%$ en milieu urbain.

Par rapport à l'accès aux soins de santé, cette étude a montré que la distance à parcourir pour arriver aux structures de santé reste très longue en milieu rural $(6$ à $50 \mathrm{~km}$ ) tandis qu'en milieu urbain, $72 \%$ d'enquêtés parcourent moins de $6 \mathrm{~km}$ pour joindre les structures de santé. Le niveau de satisfaction reste élevé en milieu urbain avec $78.1 \%$ contre $63.5 \%$ en milieu rural.

Toutefois, l'espoir est permis dans ce sens que l'Etat burundais s'est déjà doté de la Politique Nationale de la Réforme Administrative (PNRA) en conformité avec la vision 2025 et le Cadre Stratégique de Croissance et de Lutte contre la Pauvreté (CSLP).

La PNRA est donc un outil qui permet d'améliorer la qualité des services publics au Burundi. Cette politique introduit entre autres la politique de gestion des performances dans les services publics.

De manière générale, cette étude a permis de dégager des lacunes au niveau du fonctionnement des services de base en l'occurrence l'eau et la santé. Il ressort des analyses un besoin pressant pour le gouvernement d'engager des réformes profondes au niveau des différents services publics 
dans le but de satisfaire les citoyens et de développer durablement le pays. Depuis 2005, les résultats atteints sont satisfaisants mais l'action du gouvernement mérite une lecture beaucoup plus éclairée et plus prudente pour atteindre les Objectifs de Développement Durable (ODD).

\section{References:}

1. Aghion P. et Howitt P., (1992). "A Model of Growth through Creative Destruction", In Econometrica. pp. 323-351.

2. Aktouf, O. (1987). Méthodologie des sciences sociales et approche qualitative des organisations. Une introduction à la démarche classique et une critique. Université du Québec. Montréal.

3. Assogba, Y. (2008). Développement communautaire en Afrique. Comprendre la dynamique des populations. Québec : Les Presses de l'Université Laval.

4. Awomo, N.J-.C., Mahieu, P.-A., \& Tsafack, N. R. (2014). Mutuelles de santé et état de santé des populations au Cameroun : une enquête conduite dans la région du Centre pour estimer l'effet de l'adhésion à une mutuelle sur l'état de santé déclaré, in, Journal de gestion et d'économie médicale, 2014/4 (Vol. 32). Yaoundé : Université de Yaoundé II, REMA.

5. Bachelard, G. (1980). La formation de l'esprit scientifique. (10 ème éd.). Paris : Librairie philosophique J. VRIN.

6. Beaud, S. \& Webert, F. (2003). Guide de l'enquête de terrain. Paris : La Découverte.

7. Becker, G. (1994). Human Capital: A theoretical and Empirical Analysis with Special Reference to Education, University of Chicago Press, 3 Édition.

8. BIT, (2002). Micro-assurance santé. Guide d'introduction aux mutuelles de santé en Afrique. Programme Stratégies et Techniques contre 1'Exclusion sociale et la Pauvreté (STEP). Genève : BIT.

9. Brou, A. N. et al. (2018). Perceptions Sociales De L'hygiène Et De L'assainissement En Milieu Urbain Et Rural Ivoirien. In European Scientific Journal January 2018 edition Vol.14, No.2 ISSN: 1857 7881 (Print) e - ISSN 1857- 7431.

10. Boyodi Katanga, T. et al. (2016). La Chirurgie Foraine : Une Solution Aux Problèmes D'accès Aux Soins Chirurgicaux Des Populations Rurales. Macedonia: In, European Scientific Journal. December 2016 edition vol.12, No.36 ISSN: 1857 - 7881 (Print) e - ISSN 1857- 7431.

11. Charlier, J.-E. et Campenhoudt, (Sous la direction de) (2014). 4 méthodes de recherche en sciences sociales, cas pratiques pour l'Afrique francophone et le Maghreb. Paris : Dunod. 
12. Comte, A. (1970). Plan des travaux scientifiques nécessaires pour réorganiser la société. Paris: Les Éditions Aubier-Montaigne. Collection : La philosophie en poche. Publication originale : mai 1822.

13. Defourny, J. \& Failon, J. (2011). Les déterminants de l'adhésion aux mutuelles de santé en Afrique subsaharienne: un inventaire des travaux empiriques. In Mondes en développement 2011/1 ( $\left.n^{\circ} 153\right)$.

14. De Miras, C. et Le Tellier, J. (2005). Gouvernance urbaine et accès à l'eau potable au maroc, Partenariat Public-Privé à Casablanca et Tanger-Tétouan. Paris : L'Harmattan.

15. Djimouko, S. et al. (2016). L'utilisation des structures de soins dans la région du logone occidental au sud du Tchad, Un défi pour la gouvernance en santé. Macedonia: In European Scientific Journal. October 2016 edition vol.12, No.30 ISSN: 1857 - 7881 (Print) e ISSN 1857- 7431.

16. Doubogan, Y. O., Badou, S. A. O. \& Atchouta R. A. (2017). De La Politique De Santé Publique Aux Démarches D'entraide Sociale : Mutuelles De Santé Et Équité Sociale Dans Le Département Du Borgou Au Bénin. Macedonia: In European Scientific Journal. January 2017 edition vol.13, No.3 ISSN: 1857 - 7881 (Print) e - ISSN 1857- 7431.

17. Fiechter-Widemann, E. (2015). Droit humain à l'eau: justice ou...imposture? Thèse de doctorat : Univ. Genève.

18. Ghiglione, G., et Matalon, B. (1978). Les enquêtes sociologiques. Paris : Editions Armand Colin.

19. Gouvernement du Burundi, Programme National des Réformes Administratives, PNRA, 2012.

20. Groupe de la Banque Africaine de Développement, Commission de l'Union Africaine, Commission économique pour l'Afrique. (2016). Annuaire statistique pour l'Afrique 2016.

21. Guy R. (1968). Introduction à la sociologie générale. Tome 1: L'action sociale. Paris : Editions HMH, Ltée.

22. https://www.mieux-vivre-autrement.com/les-besoins-fondamentauxetre-humain.html

23. Ibrahima, K. (2015). Qualité de vie et santé dans un quartier à habitat indigne de la ville d'Abidjan (côte d'Ivoire) : rôle de l'assainissement dans ce débat écologique. Macédonia. In European Scientific Journal. October 2015 édition, vol.11, No.29 ISSN: 1857 - 7881 (Print) e ISSN 1857-7431

24. Ibrahima, S. (2017). Déterminants de l'adhésion et de la fidélisation aux mutuelles de santé dans la région de Ziguinchor (Sénégal). In Santé publique 2017/1 (Vol. 29). S.F.S.P. 
25. Institut de Statistiques et d'Etudes Economiques du Burundi (ISTEEBU), (2012). Enquête ménages pour le suivi et l'évaluation de l'impact de l'appui au système de remboursement du Paquet Minimum des Services de santé. Rapport final. Bujumbura.

26. Javeau, C. (1990). L'enquête par questionnaire. Manuel à l'Usage du

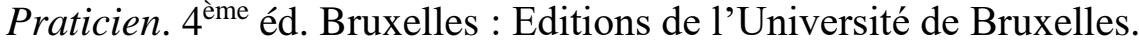

27. Médecins sans frontières (2004). Accès aux soins de santé au Burundi, résultats de trois enquêtes épidémiologiques. Bujumbura.

28. Mincer, J. (1958), "Investment in Human Capital and Personal Income Distribution." In The Journal of Political Economy, Vol. 66, No. 4, pp. 281-302.

29. Ministère de l'Education, de l'Enseignement Supérieur et de la Recherche Scientifique (MESRS), statistiques du secteur éducatif burundais, annuaire, 2012 / 2013. Bujumbura.

30. Ministère de l'Eau, de l'Environnement, de l'Aménagement du Territoire et de l'Urbanisme (MEEATU), (2013). Politique Nationale d'Assainissement du Burundi et Stratégie Opérationnelle Horizon 2025. Bujumbura.

31. Ministère de l'Eau, de l'Environnement, de l'Aménagement du Territoire et de l'Urbanisme (MEEATU). Politique nationale de l'Eau, 2012. Bujumbura.

32. Ministère de l'Eau, de l'Environnement, de l'Aménagement du Territoire et de l'Urbanisme (MEEATU). Code de l'Eau, 2012. Bujumbura.

33. Ministère de la Santé Publique et de Lutte contre le SIDA (MSPLS), (2012). Enquête sur la santé. Bujumbura.

34. Ministère de la Fonction Publique, du Travail et de l'Emploi (MFPTE), (2012). Politique nationale de gestion des performances. Bujumbura.

35. Ministère de l'Eau, de l'Environnement, de l'Aménagement du Territoire et de l'Urbanisme (MEEATU). Stratégie Nationale de l'Eau (2011-2020). Bujumbura.

36. Ministère de la Santé Publique et de Lutte Contre le SIDA (2009). Plan stratégique de la réponse du secteur de la santé face au VIH/SIDA et aux IST au Burundi pour la période 2010-2015. Bujumbura.

37. Ministère de la Santé Publique et de Lutte contre le SIDA (MSPLS), (2008). Processus d'élaboration de la politique et du plan de développement des ressources humaines. Bujumbura.

38. Ministère de la Santé Publique et de Lutte contre le SIDA (MSPLS), (2004). Politique nationale de la Santé. Bujumbura.

39. Ministère de la sante publique et de la lutte contre le sida. Plan National de Développement Sanitaire, 2011 - 2015. 
40. Ministère de la sante publique et de la lutte contre le sida (2014). Etude sur le financement de la santé au Burundi. Rapport de synthèse. Bujumbura.

41. Mondjanagni, A.-C. (1984). La participation populaire au développement en Afrique noire, in, Cahiers de l'Institut Panafricain pour le Développement (IPD) ; 23 - 49. Paris: Karthala.

42. Mucchielli, A. (1991). Les méthodes qualitatives. Paris : PUF.

43. Munyerere, B. (2015). Analyse des stratégies des jeunes dans la lutte contre le chômage au Burundi : Cas des jeunes diplômés de la zone Cibitoke. Mémoire de Licence. HAU.

44. N'da, P. (2002). Méthodologie de la recherche. De la problématique à la discussion des résultats. Comment réaliser un mémoire, une thèse en Sciences sociales et en Education. Abidjan : EDUCI.

45. N'da, P (2007). Méthodologie et guide pratique du mémoire de recherche et de la thèse de doctorat. Paris : L'Harmattan.

46. Ndabarushimana, A. (1999). Programme d'Ajustement Structurel et Appauvrissement au Burundi. Travail de Fin de Cycle (TFC). Nairobi : Université Internationale Espoir.

47. Ndabarushimana, A. (2014). Innovations techniques caféicoles et développement rural: Etude du cas de l'expérience burundaise à travers l'exemple de la fédération bonakure de la province kayanza au nord $d u$ Burundi. Thèse unique de doctorat en sociologie, option, sociologie rurale. Université Félix Houphouët Boigny d'Abidjan/Cocody. Abidjan.

48. Ndabarushimana, A., Toh, A., Kouadio, A, A. (2016). Innovations Techniques Caféicoles Au Burundi : Étude Des Contraintes SocioÉconomiques Et Des Conditions D’adoption Et De Diffusion Des Innovations Techniques Dans La Province Kayanza. Macedonia: In European Scientific Journal. June 2016 edition vol.12, No.17 ISSN: 1857 - 7881 (Print) e ISSN 1857- 7431.

49. Ndabarushimana, A. Caféiculture Et Accès Aux Soins De Santé Au Burundi: Analyse De La Dynamique Des Mutuelles De Santé Des Caféiculteurs De La Province Kayanza Au Nord Du Burundi. Macedonia: In European Scientific Journal, January 2018 edition Vol.14, No.3 ISSN: 1857 - 7881 (Print) e - ISSN 1857- 7431.

50. Observatoire Régional de l'Environnement Poitou-Charentes. (2016). L'eau en Poitou-Charentes, qu'est-ce qu'une eau potable ?

51. Observatoire de l'Action Gouvernementale (OAG). (2012). Burundi : situation économique à la veille de la célébration du cinquantième anniversaire de l'indépendance. Bujumbura.

52. OIT, (2014). Evaluation technique pour la mise en place d'un Socle de protection sociale au Burundi. Rapport au gouvernement. Bujumbura. 
53. Ouellet, A. (1987). Processus de recherche une approche systématique. Québec : Presses Universitaires de Québec.

54. PNUD, (2010). Stratégie intégrée d'Appui des Nations Unies au Burundi, 2010-2014. Consolidation de la paix et relèvement communautaire. Bujumbura.

55. Ravez, L., \& Hanson, B. (2007). L'accès aux soins de santé: un droit pour tous les êtres humains. In Ethica Clinica, (n²46), 16-22.

56. République du Burundi, CSLP I, (2007).

57. République du Burundi, Vision 2025 du Burundi, 2011.

58. République du Burundi, CSLP II, (2012).

59. République du Burundi, Plan National de Développement, (20182027).

60. République du Burundi, (2012), Plan sectoriel de développement de l'éducation et de la formation 2012-2020. Bujumbura.

61. Rist, G. (1996). Le développement. Histoire d'une croyance occidentale. Paris : HEC.

62. Romer, P. (1990). "Capital, Labor, and Productivity", In Brookings Papers on Economy Activity Microeconomies, Vol. 1990.

63. Santsa Nguefack, C. V., Ndjouenkeu, R. et Ngassoum, M. B. (2018). "Pollution De L'eau De Consommation Humaine Et Risques Sanitaires A Court Terme : Cas Du Bassin Versant De La Menoua (Ouest-Cameroun)". Macedonia: In European Scientific Journal, December edition, Vol.14, No.3 ISSN: 1857 - 7881 (Print) e - ISSN 1857- 7431, pp96-116.

64. Schultz T. P. (1961). "Investment in Human Capital," In American Economy Review, pp. 1-17.

65. Union Africaine, (1982). Charte Africaine des Droits de l'Homme et des Peuples, Addis Abeba.

66. Union Africaine, Projet SOPSA, Guide de recherche, 2015.

67. Union Africaine, Charte africaine sur les valeurs et principes $d u$ service public et de l'administration publique.

68. Ziad, T. (2017). Le droit à l'eau: Un droit fondamental des autres droits de l'homme. Macedonia. In European Scientific Journal. May 2017 edition Vol.13, No.14 ISSN: 1857 - 7881 (Print) e - ISSN 1857- 7431. 